\title{
CUESTIONES ÉTICO-JURÍDICAS EN TORNO A LA VACUNACIÓN PÚBLICA*
}

\author{
ETHICAL-LEGAL ISSUES REGARDING PUBLIC VACCINATION
}

\author{
Pol Cuadros Aguilera \\ Universitat de Lleida
}

Fecha de recepción: 3-4-20

Fecha de aceptación: 1-9-20

Resumen: A pesar de que la vacunación es el medio más eficaz para controlar y prevenir enfermedades infecciosas, el creciente auge del movimiento antivacunas ha logrado reducir el número de vacunaciones y representa, por ello, una seria amenaza para la salud pública. Ante esta situación, en este artículo se analizan los aspectos ético-jurídicos más importantes relacionados con la vacunación, y cómo deberían tenerse en cuenta a fin de configurar las políticas en la materia. Al lado de ello, se presenta el modelo de vacunación pública que mejor respondería a los problemas éticos de la vacunación, examinando la forma en la que podría desarrollarse este modelo en el contexto de nuestro Derecho.

Abstract: Despite the fact that vaccination is the most effective way to control and prevent infectious diseases, the growing boom of the anti-vaccine movement has reduced the number of vaccinations and, therefore, represents a serious threat to public health. In this situation, this article analyses the most important ethical-legal aspects related to vaccination, and how they should be taken into account in order to configure the policies on the matter. Beside this, the public vaccination model that would best respond to the ethical problems of vaccination is presented, examining the way in which this model could be developed in the context of our Law.

Palabras clave: vacunación, salud pública, ética, inmunidad de grupo Keywords: vaccination, public health, Ethics, herd immunity

* Proyecto de investigación del Ministerio de Ciencia, Innovación y Universidades, titulado "Salud Pública en Transformación: Desinformación, Alimentación y Cambio Climático, cuya referencia es PID2019-107212RA-I00.

ISSN: 1133-0937

DERECHOS Y LIBERTADES

DOI: https://doi.org/10.20318/dyl.2021.6111

Número 45, Época II, junio 2021, pp. 365-398 


\section{LA INMUNIDAD DE GRUPO Y EL RECHAZO A LA VACUNACIÓN}

Existen múltiples enfermedades infecciosas que pueden provocar serios daños, gran morbilidad, e incluso la muerte. Muchas de esas enfermedades, sin embargo, pueden prevenirse y controlarse a través de la vacunación, cuya introducción sistemática ha salvado, y continúa haciéndolo, millones de vidas, reduciendo también la morbilidad que generan y, en definitiva, contribuyendo a mejorar la salud de las personas ${ }^{1}$. De hecho, la OMS ha calculado que la inmunización a través de las vacunas evita a nivel global entre dos y tres millones de muertes al año, y que, de incrementase la cobertura vacunal mundial, se podría evitar otro millón y medio de defunciones anuales ${ }^{2}$.

Uno de los mayores triunfos alcanzados por el hombre ha sido precisamente la erradicación de la viruela en el año 1980, así como la práctica desaparición de la poliomielitis, además del sarampión y de la rubeola. Y ello se

1 Aunque la vacunación se sitúa en un terreno con gran incidencia de la Ciencia, no está en los objetivos de este artículo entrar en el proceloso terreno del conocimiento científico, por entender que su exigencia quedaría fuera de nuestro alcance, y por no correr el riesgo de desvirtuar una reflexión que pretende ser ética y jurídica. Asimismo, las vacunas objeto de este artículo son las llamadas "vacunas sistemáticas", esto es, las que tienen un interés sanitario de tipo comunitario y que se aplican a la totalidad de la población, formando parte de los programas de vacunación pública. Dentro de este grupo tenemos todas aquellas que de forma general se emplean en la infancia y que forman parte del calendario vacunal, entre las cuales están la vacuna contra la poliomielitis, difteria, tétanos, tos ferina, hepatitis $B$, meningitis por Haemophilius influenza y meningoco C, rubeola, sarampión y paperas. Con esto queremos decir que las reflexiones que aquí se contienen se refieren a las vacunas ya conocidas, es decir, aquellas respecto a las que la medicina tiene más experiencia y conocimiento, tanto de la vacuna en sí, como de la enfermedad a la que se combate. Por el contrario, no nos ocuparemos de las vacunas no sistemáticas, es decir, aquellas que no tienen un interés comunitario sino individual, estando sus indicaciones en función de factores de riesgo personales o ambientales de cada individuo, o ante la aparición de brotes epidémicos. Tampoco nos referiremos a todas las vacunas que pudieran descubrirse en un futuro, puesto que lo que aquí se dice difícilmente puede extenderse sistemáticamente respecto a todas las vacunas, especialmente a las futuras, pues cada una ha de ser entendida en su contexto epidemiológico concreto. A los efectos del presente artículo, se entenderá por "vacunación", las "medidas profilácticas tendentes a inmunizar a un individuo frente a la aparición de una concreta enfermedad". C. CIERCO, "Epidemias y Derecho Administrativo. Las posibles respuestas de la Administración en situaciones de grave riesgo sanitario para la población", Derecho y Salud, vol. 13, núm. 2, 2005, p. 231. Respecto a la clasificación de las vacunas en sistemáticas y no sistemáticas, véase A. GIL DE MIGUEL, Principios básicos de vacunación, Editorial Centro de Estudios Ramón Areces, Madrid, 2004, pp. 19-20.

2 OMS, 10 datos sobre inmunización, disponible en www.who.int/es/news-room/ facts-in-pictures/detail/immunization.

DERECHOS Y LIBERTADES

ISSN: 1133-0937

Número 45, Época II, junio 2021, pp. 365-398

DOI: https://doi.org/10.20318/dyl.2021.6111 
ha logrado a través de políticas de vacunación a gran escala; políticas que, por el éxito demostrado, han ido extendiéndose, de la mano de la OMS y de otros organismos internacionales, a los países con ingresos más bajos ${ }^{3}$.

Por todo ello, la vacunación es casi unánimemente considerada como uno de los avances más importantes en la historia de la medicina y, junto con la potabilización del agua o el sistema de saneamiento y eliminación de residuos, uno de los logros más trascendentes en materia de salud pública ${ }^{4}$.

Sin embargo, a pesar de los éxitos que acompañan a la vacunación, y de los numerosos datos empíricos a su favor arrojados por las sucesivas campañas de vacunación pública, cada vez son más las personas que deciden no vacunarse, o que no permiten que se vacune a sus hijos e hijas ${ }^{5}$. Y si bien es cierto que, desde la introducción de los primeros programas de vacunación a gran escala a principios del siglo XIX, siempre ha habido sectores de la población con una opinión negativa respecto de las vacunas, durante las últimas décadas ha habido un incremento en el cuestionamiento y rechazo de las mismas como no se había producido nunca. Y esto se debe en gran parte a que, en los países con ingresos más altos, se ha consolidado un vigoroso movimiento "antivacunas" que, a través de internet y de las redes sociales, difunde un claro mensaje contrario a la vacunación, el cual está consiguien-

3 Véase, por ejemplo, el Plan de Acción Mundial sobre Vacunas elaborado por la OMS para los años 2011-2020.

4 Sobre esto véase L. K. BALL, et al., "Risky Business: Challenge in Vaccine Risk Communication", Pediatrics, núm. 101, 1998, pp. 453-458, y CENTRE FOR DISEASE CONTROL AND PREVENTION, Ten Public Health achievements, 2011.

5 Prueba de ello es que la American Academy of Pediatrics haya señalado que el número de pediatras estadounidenses que han registrado el caso de padres se niegan a vacunar a sus hijos ha pasado de ser el 74.5\% en 2006 al 87\% en 2013. Cf. C. HOUGH-TELFORD, et al., "Vaccine Delays, Refusals, and Patient Dismissals: A Survey of Pediatricians", Pediatrics, núm. 138, vol. 3, 2016, p. 1. Lo que diferencia a las vacunas públicas de las privadas, es que las primeras están incluidas en el Calendario común de vacunación, elaborado por el Consejo Interterritorial del Sistema Nacional de Salud, órgano dependiente del Ministerio de Sanidad, y están financiadas por la Seguridad Social. Las vacunas privadas, por su parte, no están incluidas en el Calendario, ni cubiertas por la Seguridad social, y pueden adquirirse en las farmacias con prescripción médica. Son ejemplos de vacunas de este tipo las de uso selectivo para viajeros, como las del cólera, la rabia, la tuberculosis, las encefalitis centroeuropea y japonesa, las fiebres amarilla y tifoidea, la hepatitis A o la meningitis meningocócica. Para saber cuáles son las vacunas públicas, véase CONSEJO INTERTERRITORIAL. SISTEMA NACIONAL DE SALUD, Calendario común de vacunación a lo largo de toda la vida. Calendario recomendado año 2020, diciembre de 2019. disponible en https://www.mscbs.gob.es/profesionales/ saludPublica/prevPromocion/vacunaciones/docs/CalendarioVacunacion_Todalavida.pdf

ISSN: 1133-0937

DOI: https://doi.org/10.20318/dyl.2021.6111
DERECHOS Y LIBERTADES

Número 45, Época II, junio 2021, pp. 365-398 
do revertir la opinión pública que se tenía respecto a esta técnica y reducir significativamente los niveles de inmunización ${ }^{6}$.

Si este fenómeno social preocupa enormemente a las autoridades sanitarias, es porque está haciendo aparecer o, mejor dicho, reaparecer, enfermedades de las que hacía muchos años que no se tenían registros ${ }^{7}$. El problema se agrava cuando los individuos no vacunados viven concentrados en zonas o "bolsas" de población, por cuanto que favorecen la aparición de brotes epidémicos. En Europa, por ejemplo, a pesar de que el sarampión era una enfermedad prácticamente extinguida, desde 2017 ha habido un incremento de los casos, que han pasado de ser 5.000 en 2016, a ser más de 21.000, y con aproximadamente 40 fallecidos, al año siguiente. Lo preocupante, nuevamente, es que en 2017 más del 80\% de los niños y adolescentes contagiados de sarampión no habían sido vacunados, a pesar de que la vacuna de esa enfermedad estaba disponible y de forma gratuita en todos los países de la Unión Europea ${ }^{8}$.

A pesar de estos datos, la opinión pública no empezó a ver con inquietud al movimiento "antivacunas" hasta que los medios, en la segunda década del siglo XXI, se hicieron eco de una serie de casos de menores infectados con desenlace fatal, lo cual hizo que las autoridades y expertos en salud pública empezaran a preguntarse de qué modo debían hacer frente al desafío que se les planteaba. Nos referimos, por ejemplo, a casos como el del niño de

Federico de Montalvo ha señalado que, aunque en España los niveles de vacunación en 2007 no eran inferiores al 92\% en ninguna comunidad autónoma, cada vez hay una presencia más palpable en nuestro país de un movimiento que rechaza la vacunación. En este sentido, entiende que, aunque actualmente no estemos ante un conflicto de dimensiones relevantes en relación con el movimiento antivacunas en nuestro país, el Derecho ha de trabajar en su prevención, adelantándose a que determinados hechos o tendencias futuras vengan a confirmar que lo que la actualidad nos muestra hoy sea, en realidad, la punta de un iceberg. F. DE MONTALVO JÄÄSKELÄINEN, “Rechazo a las políticas públicas de vacunación. Análisis constitucional del conflicto desde los sistemas español y norteamericano", Derecho Privado y Constitución, núm. 26, 2012, pp. 215 y 234.

7 Por ejemplo, en el Estado de California, en 2010, se registró una epidemia de tosferina que fue la más importante desde 1947. En 2012 le siguió el Estado de Washington con la epidemia más importante de esa misma enfermedad desde 1942. Cf. J. E. ATWELL, et al., "NonMedical Vaccine exemptions and Pertussis in California 2010", Pediatrics, núm. 132, 2013, pp. 624-630; y CENTRE FOR DISEASE CONTROL AND PREVENTION, "Pertussis EpidemicWashington, 2012", Morbidity and Mortality Weekly Report, núm. 61, 2012, pp. 517-522.

8 A. AMMON, y X. PRATS, “Vaccines, Trust, and European Public Health", Euro Surveil, núm. 23, vol. 17, 2018, pp. 1-3; y EUROPEAN CENTRE FOR DISEASE CONTROL AND PREVENTION, Measles in the EU/EEU: Current Outbreaks, Latest Data and Trends, 2018. 
6 años de la localidad catalana de Olot, el cual en 2015 contrajo difteria sin estar vacunado por expresa voluntad de sus padres, y que terminó falleciendo ese mismo año. La noticia ganó en trascendencia porque el último caso de esa enfermedad registrado en Cataluña fue en 1983. De hecho, en 1941 se registraron en España 1.000 casos de difteria por cada 10.000 habitantes, pero tras una campaña de vacunación pública iniciada en 1947 y que se prolongó mucho en el tiempo, la enfermedad fue declarada erradicada en $1987^{9}$.

Otro caso mediático fue el del niño de 6 años que falleció por sarampión en 2017 en Italia. El menor, al que se le había diagnosticado leucemia, y al cual, por ello, la vacunación le estaba contraindicada, fue contagiado por sus dos hermanos mayores, quienes tampoco estaban vacunados en su caso por expreso deseo de la familia ${ }^{10}$. En Francia, también en 2017, falleció una joven de 17 años por sarampión que tampoco estaba vacunada, pues se había sometido a un trasplante de corazón, una causa contraindicada para vacunarse. A este caso le siguió, también en el país galo, los fallecimientos por la misma enfermedad de una paciente de 26 y otra de 32 años que tampoco habían sido vacunadas ${ }^{11}$. Por último, en los EE.UU., en donde el sarampión fue considerado erradicado en 2000, hubo un brote epidémico en el parque de atracciones de Disney en California en 2014. A principios de 2015, los infectados por el foco ocasionado en ese parque ascendía a 102, de los cuales el 40\% fueron menores de 20 años, y, entre estos, el 17\% menores de 4 años ${ }^{12}$. Hay que tener presente que padecer alguna de las enfermedades prevenibles a través de la vacuna, como el sarampión, la poliomielitis, la viruela, la meningitis o la rubeola, no sólo puede provocar la muerte, sino dejar importantes e irremediables secuelas físicas y psíquicas, que pueden generar en quien las padece, además, un estigma social que les haga sufrir discriminaciones, por ejemplo, en el ámbito laboral ${ }^{13}$.

Lo siguiente que cabría preguntarse es por qué hay individuos no vacunados, o, mejor dicho, qué razones pueden llevar a un individuo a rechazar

$9 \quad$ P. LINDE, “Quiénes son los antivacunas”, El País, 27 de junio de 2015.

10 I. MONZÓN, "Muere un niño de sarampión en Italia tras ser contagiado por sus hermanos no vacunados", El Español, 23 de junio de 2017.

11 E. CANTÓN, "Un nuevo caso de sarampión reaviva el debate de las vacunas en Francia", El Periódico de Catalunya, 11 de julio de 2018.

12 P. XIMÉNEZ, "El brote de sarampión de Disneyland supera los 100 casos en 14 estados", El País, 4 de febrero de 2015.

13 Para entender la forma en la que esta situación podría encajar en un supuesto de discriminación en el ámbito laboral, véase M. PUIG, "La tensión entre la libertad ideológica y la libertad de empresa”, Derechos y Libertades, núm. 42, 2020, pp. 305-308.

ISSN: 1133-0937

DOI: https://doi.org/10.20318/dyl.2021.6111
DERECHOS Y LIBERTADES

Número 45, Época II, junio 2021, pp. 365-398 
la vacunación o impedir que se vacune a sus hijos. Hay dos grandes motivos que explican esto. El primero de ellos tiene que ver con un acceso muy difícil o nulo a las vacunas. Si bien es el supuesto típico de los países con ingresos bajos, también se da en Europa, sobre todo en grupos de población que viven aislados o al margen de la sociedad, ya sea por razones étnicas, culturales, o incluso geográficas. Son colectivos que, a su aislacionismo, suelen añadir el desconocimiento, la desinformación, o la falta de interés por las vacunas ${ }^{14}$.

El segundo motivo se refiere ya al supuesto general de individuos que residen en países con ingresos altos y que sí tienen acceso a las vacunas. Pero dentro de este grupo, a su vez, hay que distinguir entre los individuos que, aunque pueden vacunarse, deciden no hacerlo de forma libre y consciente y aquellos otros que, aunque quieran, no pueden vacunarse por razones médicas. Veamos esto.

Empecemos por considerar las razones no médicas, esto es, las razones por las que un habitante de un país con altos ingresos, sano e informado, rechazaría la vacunación. Las más comunes de este tipo serían, en primer lugar, las que ponen en duda la seguridad y efectividad de dicha técnica ${ }^{15}$. En este sentido, se dice, por ejemplo, que las vacunas están compuestas de sustancias tóxicas o potencialmente nocivas; que provocan efectos secundarios perjudiciales, como por ejemplo el autismo, la diabetes $\mathrm{y}$, sobre todo, que debilitan el sistema inmunológico ${ }^{16}$. O bien se defiende que las vacunas no son necesarias, porque las enfermedades de las que previenen son muy poco frecuentes y están desapareciendo naturalmente ${ }^{17}$.

En segundo lugar, encontramos razones para el rechazo que se fundamentan en la creencia en métodos alternativos que previenen o reducen la incidencia de enfermedades, como la homeopatía, la toma de complementos vitamínicos, una mejor higiene, o los hábitos de vida saludables ${ }^{18}$. Incluso

14 Sobre las "poblaciones infravacunadas", véase C. CIERCO, Vacunación, libertades individuales y Derecho público. Ensayo sobre las principales claves para la regulación de la vacunación pública en España, Marcial Pons, Madrid, 2018, p. 105.

15 Sobre este tipo de razones véase P. SMITH, et al., "Parental Delay or Refusal of Vaccine Doses, Childhood Vaccination Coverage at 24 Months of Age, and the Health Belief Model", Public Health Reports, supl. 2, vol. 126, 2011, pp. 135-146.

16 M. NAVIN, et al., "Vaccine Education, Reasons for Refusal, and Vaccination Behaviour", American Journal of Preventive Medicine, núm. 56, vol. 3, 2019, pp. 359-367.

17 Puede encontrarse más información al respecto en S. BLUME, "Anti-vaccination movements and their interpretations", Social Science \& Medicine, núm. 62, 2006, pp. 628-642.

18 Véase al respecto A. KATA, “A postmodern Pandora's box: Anti-vaccination misinformation on the Internet", Vaccine, 28, 2010, pp. 1709-1716; y I. HARMSEN, et al., "Why pa- 
se asegura que, a veces, es beneficioso contraer la enfermedad para adquirir inmunización de forma "natural", quedando gracias a ello protegido para el futuro $^{19}$.

En tercer lugar, hay quien enarbola razones que tienen que ver con la desconfianza hacia la medicina "oficial", a la que se ve (demasiado) comprometida con el gobierno y con los poderes económicos, como, por ejemplo, aquellos laboratorios que hipotéticamente se beneficiarían de la extensión de las vacunas. De acuerdo con Bert Baumgaertner, por ejemplo, aquéllos que menos confían en el gobierno y en lo gubernamental en general, son más proclives a rechazar la vacunación ${ }^{20}$. Estos individuos, asimismo, prefieren informarse por su cuenta a través de internet antes que acudir a fuentes oficiales, porque no creen que la medicina institucional pueda ofrecerles un conocimiento verídico sobre la vacunación, pues sospechan que la mayor parte de los datos que se llegan a publicar por esta vía están manipulados.

En último lugar, encontramos las razones de tipo moral o religioso. Hay quien cree las vacunas son un asunto inmoral, porque para su desarrollo se experimenta con niños, y quien entiende, sencillamente, que la vacunación no es compatible con la observancia de su $\mathrm{fe}^{21}$. Es importante apuntar que las razones religiosas son las que llevan a un mayor número de personas a rechazar la vacunación en términos absolutos y son, por lo tanto, el tipo de razones no médicas más frecuentes ${ }^{22}$.

Vistas las razones de tipo no médico, a su lado encontramos razones estrictamente médicas que impiden o desaconsejan la administración de una vacuna. Así la existencia de alergias respecto a algún componente incluido en ella, o la de enfermedades que deprimen y debilitan el sistema inmunológico, como la leucemia. También si es el caso de tratamientos oncológicos, intervenciones quirúrgicas severas, o bien la aplicación a personas con órga-

rents refuse childhood vaccination: a qualitative study using online focus groups", Biomedical Centre Public Health, núm. 13, vol. 1, 2013, pp. 1-8.

19 Así lo ha recogido, por ejemplo, A. GIUBILINI en The Ethics of Vaccination, Palgrave Macmillan, Londres, 2019, p. 14.

20 B. BAUMGAERTNER, et al., "The influence of political ideology and trust on willingness to vaccine", en PLOS One, núm. 13, vol. 1, 2018, p. 9.

21 Merece la pena señalar que, aún lo paradójico que resultan algunos motivos aducidos por quienes rechazan la vacunación, por ejemplo el citado de que para el desarrollo de vacunas se experimenta con niños, lo que aquí nos interesa es tan sólo presentar los motivos que alegan quienes se oponen a la vacunación, pero no averiguar su veracidad. Sobre esto, véase A. KATA, “A postmodern Pandora's box...”, cit., p. 1710.

22 OMS, Report of the SAGE Working Group On Vaccine Hesitancy, 2014, p. 18.

ISSN: 1133-0937

DOI: https://doi.org/10.20318/dyl.2021.6111
DERECHOS Y LIBERTADES

Número 45, Época II, junio 2021, pp. 365-398 
nos trasplantados, o a recién nacidos, demasiado débiles aún para ser inmunizados, etcétera. Son ejemplos, en definitiva, de incompatibilidad entre el estado de salud del individuo y la administración de la vacuna ${ }^{23}$.

¿Significa esto que las personas que padecen alguna de estas contraindicaciones quedarían plenamente desprotegidas y expuestas a contraer enfermedades infecciosas? Lo cierto es que no. Existen dos modos de "inmunizarse" frente a enfermedades víricas a los que pueden recurrir los individuos, dependiendo de la situación médica en la que se encuentren. Por un lado encontramos la vacunación, que es el modo más eficaz, directo y preferente de inmunización y, por el otro, la llamada "herd immunity", o "inmunidad de grupo", cuya protección funciona cuando una alta proporción de la población es inmune, por ejemplo porque ha sido vacunada contra una determinada enfermedad y, en consecuencia, el virus no puede seguir transmitiéndose de persona en persona, ¡ni siquiera a los no vacunados!, por lo que su circulación se interrumpe, y la enfermedad termina por erradicarse ${ }^{24}$.

Pero para blandir satisfactoriamente el escudo de la inmunidad de grupo es necesario, en muchos casos, alcanzar una altísima cobertura vacunal, que generalmente se encuentra entre el 90 y el 95\% de la población, aunque esta cifra sea variable y dependa de la enfermedad concreta. Así, se estima necesaria una cobertura del $92-95 \%$ para alcanzar la inmunidad de grupo respecto al sarampión, el $92-95 \%$ para la tosferina, el $90-92 \%$ para las paperas, el $85-97 \%$ para la rubeola, el $80-85 \%$ para la difteria, y el $80-85 \%$ para la poliomielitis ${ }^{25}$.

En cuanto a su naturaleza social, Angus Dawson considera que la inmunidad de grupo tiene las características propias de un bien público, por cuanto que no genera exclusión ("non-excludable") ni rivalidad ("non-rivalrous") en quienes se benefician de él ${ }^{26}$. Mientras que, en principio, un bien

23 JESSICA FLANIGAN da ejemplos de los peligros a los que se exponen los individuos no vacunados por razones médicas en "A Defence of Compulsory Vaccination", HealthCare Ethics Committee Forum, núm. 26, vol. 5, 2014, p. 9.

24 Podemos encontrar otro tratamiento de la "inmunidad de grupo" desde un punto de vista ético o social en R. E. SPIER, "Ethical aspects of vaccines and vaccination", Vaccines, vol. 16, núm. 19, pp. 1788-1794, y en J. SCHWARTZ, y A. CAPLAN, "Ethics of vaccination programs", Current Opinion in Virology 1, 2011, pp. 263-267.

25 Estos datos han sido obtenidos en R. M. ANDERSON, “The concept of herd immunity and the design of community-based immunization programmes", Vaccine, vol. 10, núm. 13, 1992, p. 929.

26 A. DAWSON, "Herd Protection as a Public Good: Vaccination and our Obligations to Others", A. DAWSON, y M. VERWEIJ, (editors), Ethics, Prevention, and Public Health, Oxford University Press, 2007, p. 163-164. 
privado sólo genera efectos positivos inmediatos en quien goza de él, la inmunidad de grupo, en tanto que bien público, produce beneficios para todos los miembros de la comunidad. Por eso se dice que es "non-excludable", es decir, que no puede excluir con facilidad a individuos concretos respecto a los beneficios que genera, incluso cuando haya quien no quiera beneficiarse, o no haya contribuido a su consecución. Por último, es "non-rivalrous", por cuanto que no exige que los individuos compitan o rivalicen entre sí para sacar provecho del bien. Otros bienes que comparten estas características son, por ejemplo, el aire puro, la defensa nacional, los diques de protección frente a inundaciones, o la salubridad de las aguas.

Respecto al modo en que la inmunidad de grupo puede alcanzarse y mantenerse es importante notar, tal como recuerda Dawson, que es necesaria "the cooperation of a sufficiency large number of people", o, lo que es lo mismo, que "no individual or small group of individuals (respecto a la comunidad) can realize herd immunity" 27 . Porque sólo puede ser generada a través de una labor colectiva muy amplia, y nunca por acciones individuales o parciales, la inmunidad de grupo es un bien que debe ser visto necesaria y técnicamente como una empresa colectiva o comunitaria.

La importancia de la inmunidad de grupo, que es el bien que proporciona la vacunación obligatoria, descansa en los beneficios que reporta, los cuales, de acuerdo con Alberto Giubilini, quien ha dedicado al tema varios capítulos de su libro The Ethics of Vaccination, son fundamentalmente tres. En primer lugar, la protección de los individuos no vacunados (no debe olvidarse sin embargo que, tal como advierte este autor, la inmunidad de grupo, aun constituyendo la mejor manera para proteger a los que no pueden vacunarse por razones médicas, no ofrece el mismo nivel de protección que la vacunación individual, y que, por lo tanto, no debe ser vista como una alternativa equivalente a la vacunación) ${ }^{28}$. En segundo lugar, la protección de los propios vacunados, ya que ninguna vacuna es $100 \%$ efectiva ${ }^{29}$. Por último, el considerable ahorro en gasto público que proporciona, al evitar los gastos de tratar a los pacientes de enfermedades prevenibles a través de la vacunación

27 Ibidem.

28 A. GIUBILINI, The Ethics of Vaccination, cit., p. 19.

29 El Center for Disease Control and Prevention señaló, en 2017, que la vacuna contra la tosferina sólo es un 70\% efectiva durante el primer año, y que luego decrece hasta el 30-40\% en los cuatro años siguientes a su administración. Véase CDC, Pertussis FAQ, disponible en www.cdc.gov/pertussis/about/faqs.html 
y redirigir recursos públicos a la satisfacción de otras necesidades, sanitarias o no ${ }^{30}$.

La vacuna debe ser vista en consecuencia como un recurso que no sólo tiene potenciales beneficios para el individuo al que se le administra, sino que repercute de forma positiva en la colectividad en general y en los que no pueden vacunarse en particular. Es precisamente esta repercusión en los demás lo que permite hablar de los aspectos éticos de la vacuna y la convierte en un acto, en suma, relacionado con la solidaridad.

\section{LA JUSTIFICACIÓN DE LA VACUNACIÓN OBLIGATORIA}

Al igual que la donación de sangre, la vacuna puede ser contemplada como un acto solidario y un deber moral, desde el momento en que no sólo permite proteger a los individuos vacunados, sino también a los demás, y, en especial, a aquellos individuos vulnerables que no pueden vacunarse por razones médicas y que ven en la vacunación de sus conciudadanos la mejor salvaguarda frente a determinadas enfermedades contagiosas. Pero si, tal como hemos visto, es justamente la protección de los demás lo que convierte la vacunación en un asunto con una dimensión ética, el modelo de vacunación pública que se adopte ha de tener en cuenta esta dimensión ética. Veamos a continuación como la tienen en cuenta diferentes modelos.

Hay que partir de la idea de que hay estados de salud que desaconsejan la vacunación, y que quienes se encuentran en ellos no tienen mejor protección que vivir en una comunidad en la que esté activada la inmunidad de grupo. Puede entenderse, por lo tanto, que esos individuos tengan derecho a reclamar protección frente a enfermedades infecciosas exigiendo a sus conciudadanos que contribuyan a alcanzar y mantener la inmunidad de grupo. Según Giubilini, "we have a prima facie claim not to be harmed by others, which generates a prima facie obligation on others not to harm us", o, lo que es lo mismo, existe "a prima facie claim right not to be infected by vaccine-preventable disease, when this can be achieved through vaccinations" ${ }^{31}$. Se trata de algo muy parecido a pedir a los demás su contribución para obtener bienes colectivos relacionados con la salud pública, como el aire

30 Giubilini señala, por ejemplo, que el costo total anual ocasionado para tratar a enfermos de gripe en los Estados Unidos gira en torno a los 87.1 millones de dólares. A. GIUBILINI, The Ethics of Vaccination, cit., p. 27.

$31 \quad$ Ibid., pp. 34 y 37. 
puro, el agua potable, o un sistema eficaz de saneamiento y alcantarillado, ejemplos típicos de bienes que generan en las personas ciertas obligaciones individuales ${ }^{32}$.

Si la comunidad tiene el deber de proteger a sus integrantes a través de la inmunidad de grupo, la vacunación -único medio capaz para alcanzarla- se convierte así en una obligación y en una responsabilidad comunitaria. Y esto significa, a su vez, que cada uno de los individuos que conforman la comunidad tiene el deber moral de contribuir a su realización, o lo que es lo mismo, tiene el deber moral de vacunarse. En este sentido, Angus Dawson ha asegurado que "individuals may under a moral obligation to vaccine themselves to ensure that others are not put at increased risk of harm due to their omission" ${ }^{33}$. También Giubilini cree los individuos están impelidos a vacunarse por un deber moral cuando afirma que "each and every individual member of the collective with the power to realize herd immunity has a moral obligation to contribute to the realization of the collective effect" 34 .

Es importante darnos cuenta, para comprender mejor lo que seguirá, que a través de afirmaciones como estas estamos pasando de una obligación colectiva a una obligación individual. La comunidad, entendida como el conjunto de sus integrantes, tiene el deber y la responsabilidad de proteger a sus miembros, especialmente a aquellos que no pueden hacerlo por su cuenta, y que dependen de los demás, es decir, que la comunidad tiene el deber de alcanzar la inmunidad de grupo ${ }^{35}$. Pero, al tratarse de un bien colectivo, la inmunidad de grupo supone para su consecución la atribución de ciertas obligaciones al individuo. La obligación colectiva se vuelve también individual. En palabras de nuevo de Giubilini, "this type of collective obligation entails for attribution of individual obligations to be vaccinated", o lo que es

32 Así lo cree también Giubilini, el cual asegura que "collective obligations to realize herd immunity give rise, by their very own nature, to individual obligations to contribute to herd immunity by being vaccinated". Ibid., p. 48.

33 A. DAWSON, "Herd protection as a public Good...", cit., p. 171.

34 A. GIUBILINI, The Ethics of Vaccination, cit., p. 44.

35 De hecho, el propio Comité de Bioética de España, en un documento dirigido al caso expreso de la vacunación pública, nos recuerda que de la salud pública se deriva un verdadero derecho-deber, de manera que los ciudadanos tendríamos el derecho a la protección de nuestra salud, pero al mismo tiempo seríamos también destinatarios del deber legal de someternos a dichas medidas para la debida protección de la salud pública como interés colectivo. COMITÉ DE BIOÉTICA DE ESPAÑA, Cuestiones ético-legales del rechazo a las vacunas y propuestas para un debate necesario, 19 de enero de 2016, p. 12.

ISSN: 1133-0937

DOI: https://doi.org/10.20318/dyl.2021.6111
DERECHOS Y LIBERTADES

Número 45, Época II, junio 2021, pp. 365-398 
lo mismo, "translates into an individual obligation to be vaccinated to (...) all members of a collective" ${ }^{36}$.

No es esto algo demasiado sorprendente. El alcance y mantenimiento de bienes colectivos genera cargas, y todo derecho lleva aparejado un deber. Para que un ciudadano disfrute de un derecho, alguien deberá soportar la carga que la prestación de ese derecho lleva aparejada. Ya Hans Kelsen, señaló rotundo que el derecho de uno supone la obligación de otro ${ }^{37}$, lo que Juan Ramón Capella resumió en la fórmula que dice que alguien tiene un derecho si y solo si los demás tienen deberes a su respecto (esto es, deberes acerca de aquello que se trata de proteger con cualquier noción determinada de derecho) ${ }^{38}$. En el caso que nos ocupa, para que el derecho a la protección de enfermedades prevenibles a través de la vacunación no quede vacío de contenido, aquellos que sí pueden vacunarse deben de soportar la carga de tener que hacerlo ${ }^{39}$.

La siguiente pregunta que se nos abre es: ¿En base a qué criterios podemos saber a quién corresponde el deber de soportar las cargas que genera la consecución de la inmunidad de grupo? Un criterio es el que suministra el brocardo qui sentit commodum sentire debet incomodum, o lo que es lo mismo: deberán soportar las cargas que exija el mantenimiento de un bien, aquellos que se beneficien del mismo. En este mismo sentido, Dawson considera que "where individuals drive benefits from participation in a society, group or population, justice requires that they should contribute a fair share of the effort or risks that it might be necessary to run to achieve that benefit" ${ }^{\prime 4}$. En parecidas y famosas palabras se pronunció John Stuart Mill cuando defendió que todo aquel que recibe la protección de la sociedad tiene que devolver algo a cambio de este beneficio, y el hecho de vivir en sociedad hace indispensable que cada uno esté obligado a observar una cierta línea de conducta hacia los demás. A lo que añade que esa conducta consiste en que "cada persona asuma su parte (...) de los trabajos y sacrificios necesarios para defender a la sociedad o a sus miembros de daños y abusos" ${ }^{41}$.

36 Ibid., p. 44.

37 H. KELSEN, Teoría pura del derecho, Eudeba, Buenos Aires, 1973, capítulo VIII, p. 116.

38 J. R. CAPELLA, “Derechos, deberes: la cuestión del método de análisis" en J.A. ESTÉVEZ, (ed.), El libro de los deberes. Las debilidades e insuficiencias de la estrategia de los derechos, Trotta, Madrid, 2013, p. 46.

39 Sobre "la constitucionalización de la solidaridad", o la relación de la solidaridad con los deberes constitucionales, véase F. J. ANSUÁTEGUI, “Solidaridad, deberes y constitución: algunos apuntes conceptuales", Diritto Constituzionale, núm. 2, 2019, pp. 11-35.

40 A. DAWSON, "Herd protection as a public Good...", cit., p. 174.

41 J. S. MILL, Sobre la libertad, Akal, Madrid, 2014, p. 85. 
Beneficiarse de los bienes y de la comodidad que brinda la vida en sociedad, sí, pero también contribuir a su mantenimiento, y defender y proteger a la comunidad de los males que la amenazan a través del trabajo y del sacrificio, si es necesario. Ahora bien, pudiera ocurrir que determinados individuos, para los que la vacunación no estaría contraindicada por razones médicas, decidieran no vacunarse. Amparados por alguna de las razones vistas anteriormente, o por ninguna, deciden no participar en el logro y mantenimiento de la inmunidad de grupo de la que, indudablemente, se beneficiarán. Tales individuos, descritos como "free riders", son los que Mariëtte van der Hoven, en un artículo con un título tan descriptivo como "Why One Should Do One's Bit", caracteriza como aquellos que "take advantage of the efforts others have made to establish some collective good without actually contributing oneself" 42 ; o como dice Tim Dare refiriéndose a la vacunación, "unimmunized are 'free-riders' in the sense that they take the benefit of high immunization rates without exposing themselves to the risk unavoidably attendant upon attaining those rates" 43 .

El suyo sería un caso parecido al del pescador que no quiere colaborar junto con el resto de sus compañeros en la limpieza y mantenimiento de un lago, aunque vaya a sacar el mismo partido que ellos de unas aguas limpias y el consiguiente incremento de las capturas ${ }^{44}$. Es más que probable pensar que el resto de pescadores intentará convencerle para que colabore, explicándole los beneficios que le reportará el que las aguas estén limpias. Pero, si decide mantenerse en sus trece, poco más podrán hacer que reprocharle que su conducta es egoísta y poco ética, o injusta, tal y como considera van der Hoven, cuando afirma que "the unfairness of free riding lies not in the deliberate choice of agents to enjoy a benefit, but in the unwillingness to pay for that good" 45 .

Sin embargo, este ejemplo no es del todo análogo al que nos está ocupando, pues, aunque el pescador decline participar en las labores de limpieza, no por ello impedirá al resto de pescadores alcanzar el propósito de tener las

42 M. VAN DER HOVEN, “Why One Should Do One's Bit: Thinking About Free Riding in the Context of Public Health Ethics", Public Health Ethics, vol. 5, núm. 2, 2012, p. 155.

43 T. DARE, “Mass Immunization Programmes: Some Philosophical Issues”, Bioethics, vol. 12, núm. 2, 1998, p. 145.

${ }_{44}$ El primer ejemplo ha sido acuñado por GARETT CULLITY, que se refiere al "recalcitrant fisherman", y que aparece en su artículo "Moral Free Riding", Philosophy and Public Affairs, núm. 1, 1995, pp.3-34.

45 M. VAN DER HOVEN, “Why One Should Do One's Bit...”, cit., p. 159.

ISSN: 1133-0937

DOI: https://doi.org/10.20318/dyl.2021.6111
DERECHOS Y LIBERTADES

Número 45, Época II, junio 2021, pp. 365-398 
aguas limpias. Puede no ser necesaria la concurrencia del pescador recalcitrante -ni siquiera la de la mayoría de ellos- para conseguir el objetivo. Pero, en el caso de la inmunidad de grupo, sí que sería necesaria la participación del pescador, porque sería preciso vacunar a una alta proporción de la población para conseguirla.

La obligación colectiva de alcanzar la inmunidad de grupo no puede permitirse abusar del privilegio de dejar a la libre voluntad de cada individuo vacunarse o no, porque, si lo hace, un número significativamente alto de individuos puede no vacunarse, y entonces el objetivo pretendido nunca podrá ser alcanzado y el resto de contribuciones individuales resultarán estériles.

Volvamos por eso un momento a aquel lago. Supongamos que los pescadores están utilizando una pintura o un barniz para sus embarcaciones que está acabando con la pesca del lago debido a su toxicidad. Cuando los pescadores se dan cuenta, deciden inmediatamente dejar de utilizar ese producto. Todos lo hacen salvo uno. Y aunque los demás intenten persuadirle a través de la mejor pedagogía, el pescador no quiere atender a razones, por lo que los peces siguen muriendo envenenados. Esta situación es más análoga a lo que ocurriría con la inmunidad de grupo si algunos dejaran de vacunarse, por cuanto que, si se respetara su decisión -a pesar de haberles intentado convencer a través de la más exquisita pedagogía-, no sería posible alcanzarla ni mantenerla, y el resto de contribuciones individuales no serviría para nada.

El escenario que cabría explorar ahora es el de si debería tratarse a los que rechazan la vacunación de igual modo que en el primer ejemplo se trata a los pescadores recalcitrantes, es decir, si deberíamos respetar la voluntad de los individuos que no quieran vacunarse, o por el contrario deberíamos obligarles a que efectivamente lo hicieran ${ }^{46}$. Asimismo, había que responder

46 En este sentido, el principio de daño, tal y como lo entendió John Stuart Mill, nos ofrecería un argumento adicional para responder a la pregunta de si deberíamos obligar a los individuos que se negaran a vacunarse. Mill señala que el único propósito por el que puede ser ejercido legítimamente el poder sobre un miembro de una comunidad civilizada, en contra de su voluntad, es para prevenir del daño a otros. Pues bien, tal y como hemos visto, siendo la inmunidad de grupo un bien público o colectivo capaz de proteger a las personas de ciertas enfermedades, el principio de daño legitimaría optar por un modelo de vacunación pública obligatoria a fin de lograr y preservar niveles de vacunación suficientes para activar la inmunidad de grupo y proteger, de este modo, a aquellos individuos que, por ejemplo, no pueden vacunarse por razones médicas. Véase J. S. MILL, Sobre la libertad, cit., p. 35. 
a la cuestión de a quién debería corresponder semejante tarea. Se diría que es deber y responsabilidad de los poderes públicos, en tanto que garantes de la salud pública, asegurar el alcance y mantenimiento de la inmunidad de grupo, y que ello debería hacerse bien prohibiendo el uso de barnices tóxicos en un caso, bien adoptando el modelo de vacunación obligatoria en el otro ${ }^{47}$.

No parece difícil encontrar apoyo en el Ordenamiento jurídico. El artículo 43.2 de la Constitución establece que "compete a los poderes públicos organizar y tutelar la salud pública a través de medidas preventivas", y que "la Ley establecerá los derechos y deberes de todos al respecto" ${ }^{48}$. El artículo 3.1 de la Ley General de Sanidad, señala "la promoción de la salud" y "la prevención de enfermedades" como principios que deberán regir el sistema público de salud $^{49}$. El artículo 11 de la Ley de cohesión y calidad del Sistema Nacional de Salud dice que se entenderá por prestaciones de salud pública "el conjunto de iniciativas organizadas por las Administraciones públicas para preservar, proteger y promover la salud de la población", y añade que deberán considerarse como prestaciones de ese tipo "la prevención de las enfermedades, discapacidades y lesiones" ${ }^{50}$. Por su parte, el artículo 168 del TFUE señala que "la acción de la Unión (...) se encaminará a mejorar la salud pública, prevenir las enfermedades humanas y evitar las fuentes de peligro para la salud pública y psíquica". Por último, el artículo 35 de la Carta de los Derechos Fundamentales de la Unión Europea establece que "toda persona tiene derecho a acceder a la prevención sanitaria" ${ }^{51}$.

"Prevención de enfermedades", "prevención sanitaria" y "medidas preventivas"; "poderes públicos", "Administraciones públicas", "sistema público de salud" y "acción de la Unión". En definitiva, que el Estado tiene la responsabilidad y la obligación legal de tutelar la salud pública en lo que se refiere a la prevención de enfermedades. No hace falta, claro está, que el legislador explicite cuáles son los medios apropiados para ello, ni cuál es

47 Roland Pierik sostiene que la salvaguarda de la salud pública legitimaría al Estado a adoptar el modelo de la obligatoriedad por su idoneidad como recurso para asegurar la inmunidad de grupo, cuando sostiene que "compulsory vaccination is justified by the fact that the state has an obligation to guard common good of herd immunity in order to protect vulnerable people". R. PIERIK, "Mandatory Vaccination: An Unqualified Defence", Journal of Applied Philosophy, vol. 35, núm. 2, 2018, p. 386.

48 La cursiva que acompaña a la cita de esta norma, y a la de las siguientes, son nuestras.

49 Ley 14/1986, de 25 de abril, General de Sanidad.

50 Ley 16/2003, de 28 de mayo, de cohesión y calidad del Sistema Nacional de Salud.

51 Carta de los Derechos Fundamentales de la Unión Europea, proclamada por el Parlamento Europeo, el Consejo y la Comisión el 7 de diciembre del año 2000 en Niza, 2000/C, 364/01. 
la mejor forma de hacerlo. Entendemos que las vacunas son, esencialmente, medios para prevenir enfermedades, y que precisamente corresponde al Estado proteger de las enfermedades infecciosas a los individuos en general, $y$ a quienes no puedan hacerlo por ellos mismos en particular. "State has the moral responsibility to protect and promote individuals' health, especially that of the most vulnerable people (such as those who cannot be vaccinated", nos recuerda Giubilini en su trabajo sobre este asunto ${ }^{52}$.

Parece claro en consecuencia que, en relación con la inmunidad de grupo, la responsabilidad individual de vacunarse se traduciría en responsabilidad institucional de asegurar que los individuos efectivamente se vacunen. Y que, por lo tanto, correspondería al Estado imponer esa obligación, adoptando el modelo de vacunación pública obligatoria. Estaría más que justificado, en definitiva, juridificar la obligación moral de vacunarse -obligación que ya tienen los individuos de forma independiente-, y convertirla en una verdadera obligación legal.

\section{LA ADECUACIÓN CONSTITUCIONAL DE LA VACUNACIÓN OBLIGATORIA}

Acabamos de ver que, en primer lugar, puede sostenerse que la comunidad tiene la obligación de lograr la inmunidad de grupo; en segundo lugar, que esa obligación es, a su vez, individual, puesto que cada miembro de la comunidad es responsable de cumplir con la obligación de vacunarse y, en tercer lugar, que es responsabilidad del Estado asegurar que los individuos cumplen con esa obligación, a través de políticas encaminadas a alcanzar la inmunidad de grupo. Por último, que esas políticas deberían concretarse en la adopción de un modelo de vacunación pública obligatoria. Lo que vamos a ver a continuación es cómo debería desarrollarse ese modelo para encajar en un régimen de derechos y libertades como el reconocido en nuestro Ordenamiento jurídico.

Cuando los poderes públicos deben adoptar cualquier política que sirva como medio para conseguir un fin, todo lo deseable que sea este, deben respetarse una serie de principios que inspiran al Ordenamiento jurídico, como, por ejemplo, el de medida menos restrictiva, que es aquel principio que exige que la medida en cuestión sea la que menos afecte a los derechos y libertades de la ciudadanía. Debemos comprobar ahora, por lo tanto, si la vacunación

52 A. GIUBILINI, The Ethics of Vaccination, cit., p. 54. 
obligatoria, aunque legítima, sería la medida menos restrictiva para alcanzar el objetivo de la inmunidad de grupo.

A simple vista, podría parecer evidente que la vacunación obligatoria es una medida muy restrictiva con la autonomía individual, y que de haber otros medios menos intrusivos que igualmente permitieran conseguir altos niveles de inmunidad, el modelo de la obligatoriedad debería descartarse. Veamos si esto es así, y qué medidas permitirían, a priori, asegurar la inmunidad de grupo, siguiendo el orden de menos a más restrictivas.

En primer lugar, encontraríamos la promoción, el fomento, o la recomendación de la vacunación. Campañas de información o de concienciación serían ejemplos de medidas de este tipo. Sin embargo, aunque indudablemente podrían funcionar para algunos individuos, probablemente no sería un recurso eficaz en relación a aquellos otros que niegan las bondades de la vacuna, o que la rechazan categóricamente, por razones religiosas, filosóficas o de otra índole.

En segundo lugar, estaría el "nudging", un método de persuasión que prevé dirigir la conducta de los individuos empujándoles a base de pequeños estímulos. Un ejemplo típico de esta práctica es, en relación con el fomento de una dieta sana, colocar en las estanterías de los supermercados los productos más y menos saludables de forma tal que al consumidor le resulte más fácil alcanzar los primeros que los segundos. Lo particular del "nudging", por lo tanto, es que es un modo de alterar el comportamiento del individuo, pero sin llegar a prohibirle ninguna de las opciones de conducta que se le presentan ${ }^{53}$. En el caso que nos ocupa, este tipo de medidas podrían servir para aquellos que tengan razones débiles para no vacunarse, como los que alegan falta de tiempo, excesiva distancia entre su residencia y la consulta del médico, o los que no quieren afrontar los gastos inherentes a la visita médica, por ejemplo. Ahora bien, no parece que este método pueda servir para convencer, hacer cambiar de opinión, o influir en el comportamiento de aquellos que tengan "razones fuertes", como de conciencia o religiosas, para no vacunarse ${ }^{54}$.

53 Así lo creen Thaler y Sunstein, cuando definen el nudging como el modo de establecer o configurar el rango de opciones entre las que podrá escoger un individuo, de tal manera que "alters people's behaviour in a predictable way without forbidding any option or significantly changing their economic incentives". Veáse R. THALER y C. SUNSTEIN, Nudge. Improving Decisions About Health, Wealth, and Happiness, Penguin, Londres, 2008, p. 6.

54 También creen que los nudges son insuficientes para que accedan a vacunarse individuos que rechazan este método profiláctico por razones de conciencia, religiosas o filosóficas Y. SAGHAI "Salving the Concept of nudge", Journal of Medical Ethics, núm. 39, vol. 8, 2013, p. 489, y A. GIUBILINI, The Ethics of Vaccination, cit., p. 76. 
En tercer lugar, estarían los incentivos fiscales: premiar a los que acuerden vacunarse con un importe pecuniario. Se trataría de una medida con la que el poder político recompensaría una conducta que estima como deseada. Aunque podría ser efectiva, la medida estaría limitada por la situación de dependencia económica de cada individuo y por el contexto socioeconómico en general, lo que impediría que su eficacia pudiera ser conocida a priori. Además, y como casi siempre que interviene una variable económica para dirigir la conducta de los individuos, este tipo de medidas suelen ser más efectivas cuanto más bajas son las rentas de los destinatarios, y menos efectivas cuanto mayores son.

En cuarto lugar, encontramos los desincentivos asociados a la negativa de vacunarse, los cuales se podrían configurar de varias maneras. Una de ellas sería a través de la retención de ciertos beneficios fiscales. Un ejemplo de ello lo suministra la experiencia australiana, que llama "no jab, no pay" a la retención de los beneficios financieros que el gobierno destina a los padres con hijos recién nacidos $^{55}$. Otro ejemplo sería impedir que las personas no vacunadas accedieran a determinados espacios, decretar que no pudieran ingresar a ciertas categorías profesionales, como la de funcionario, o privarles del acceso a guarderías y centros educativos. Esta medida es la que se conoce como "vacuna-condición", e implica dar a la vacuna la categoría de requisito administrativo para el acceso a determinados bienes o servicios. El ejemplo paradigmático de este tipo de medidas es la multa, esto es, penalizar a los no vacunados con la imposición de un pago pecuniario, cuyo valor como desincentivo está fuera de dudas.

Aunque es innegable que los desincentivos influirían sobre la voluntad de los individuos, y que de adoptarse harían que muchos se vacunarían, el problema es que se parecen demasiado poco a una medida compatible con un modelo de vacunación voluntaria, y mucho a la típica sanción que seguiría al incumplimiento de una obligación legal. Es decir, parece que son un recurso que presupone la existencia de una obligación legal de vacunarse. Resulta paradójico establecer medidas destinadas a que la ciudadanía se vacunen de forma voluntaria que sean, en sí mismas, más coercitivas que las propias sanciones derivadas del incumplimiento de una obligación legal. Creemos, por lo tanto, que este tipo de medidas no encajan del todo bien en un sistema de vacunación "voluntario".

55 Para conocer más sobre la medida "no jab, no pay" del Gobierno australiano, véase F. H. BEARD, "No Jab, No Pay and vaccine refusal in Austalia: the jury is out", The Medical Journal of Australia, núm. 206, vol. 9, 2017, pp. 381-383. 
La conclusión que podemos extraer de todo esto es que ninguna de las medidas citadas permitiría alcanzar el objetivo de conseguir que prácticamente todas las personas accedieran a vacunarse por su propia voluntad, bien porque no son lo suficientemente coercitivas, bien porque demandan la existencia de una obligación legal de vacunarse. Así pues, descartadas las anteriores, cabe aceptar que el modelo de la obligatoriedad respetaría el principio de medida menos restrictiva, puesto que sería el único modo de conseguir que todos los individuos se vacunasen, precisamente por no depender de la capacidad de persuasión de la Administración ni, en definitiva, de la voluntad de los individuos ${ }^{56}$.

Llegados a este punto, lo siguiente que deberíamos comprobar es si los poderes públicos efectivamente están habilitados para establecer obligaciones en materia de salud pública en lo que se refiere a la vacunación. Ya comprobamos, ciertamente, que recae sobre la Administración la responsabilidad de garantizar la inmunidad de grupo. De lo que se trata ahora es de ver si el Estado tiene la capacidad técnica para generar la obligación legal que permitiría, en este caso, alcanzar dicho objetivo.

Lo primero que hay que considerar es que el artículo 43.2 de la Constitución dispone que, en lo que respecta a la tutela de la salud pública a través de medidas preventivas, "la Ley establecerá los derechos y deberes de todos al respecto". La Ley General de Sanidad, por su parte, señala, en su artículo 11, que los ciudadanos tienen el deber de cumplir con las prescripciones generales de naturaleza sanitaria comunes a toda la población, así como las específicas determinadas por los servicios sanitarios. Por último, la Ley 22/1980, en su artículo único, establece que "las vacunas contra la viruela y la difteria y contra las infecciones tíficas y paratíficas, podrán ser declaradas obligatorias por el Gobierno (...). En todas las demás infecciones (...) podrán ser recomendadas y, en su caso, impuestas por las autoridades sanitarias" ${ }^{57}$.

Lo que cabe extraer de estas disposiciones normativas es que efectivamente los poderes públicos están habilitados para establecer obligaciones en

56 Es importante señalar que también el Comité de Bioética de España recomendó el recurso de la vacunación obligatoria precisamente cuando hubiera indicios de una reducción de las tasas de vacunación que pusieran en peligro el efecto rebaño, pero que ello no debería impedir que la Administración siguiera impulsando medidas de educación, información y promoción de la vacunación. Véase COMITÉ DE BIOÉTICA DE ESPAÑA, Cuestiones éticolegales del rechazo a las vacunas y propuestas para un debate necesario, 19 de enero de 2016, p. 27.

57 Ley 22/1980, de 24 de abril, de modificación de la base IV de la Ley de Bases de la Sanidad Nacional de 25 de noviembre de 1944.

ISSN: 1133-0937

DOI: https://doi.org/10.20318/dyl.2021.6111
DERECHOS Y LIBERTADES

Número 45, Época II, junio 2021, pp. 365-398 
materia de salud pública en general, y también en concreto para tutelarla “a través de medidas preventivas". ¿Y qué medidas hay más preventivas que las vacunas? Asimismo, y más explícitamente, la Ley 22/1980 deja la puerta abierta a que el Gobierno, en caso de estimarlo oportuno, imponga la vacunación de forma obligatoria.

Ahora bien, visto esto, también hay que señalar que la normativa vigente podría no ser suficiente para desarrollar un sistema obligatorio de vacunación pública, aunque sí lo sería para imponer vacunaciones de forma puntual en caso de concurrir situaciones extraordinarias y urgentes ${ }^{58}$. De hecho, cuando la Constitución establece el mandato a los poderes públicos que conocemos, precisa que, en todo caso, los derechos y deberes sean establecidos mediante norma con rango de ley, la cual, por el momento, en lo que se refiere a las vacunas, no encontramos en nuestro Derecho ${ }^{59}$.

No obstante, ¿ha habido alguna experiencia histórica de vacunación obligatoria en nuestro país? Sí. En 1903 se sentaron las bases de la vacunación pública en España, siguiendo el modelo de la obligatoriedad, a través del Real Decreto sobre vacunación obligatoria y medios de extinción de la viruela, en cuyo artículo $6^{\circ}$ se establecía de forma categórica que "será absolutamente obligatoria la vacunación y revacunación" ${ }^{60}$. Sin embargo, este modelo no llegó a consolidarse hasta 1944, cuando la Ley de Bases de la Sanidad Nacional declaró "obligatorias las vacunaciones contra la viruela y la difteria en la forma que determinarán los reglamentos" ${ }^{61}$. Respecto al resto de vacunas, recordó que "se mantiene la obligatoriedad de las vacunaciones preventivas contra las infecciones tíficas y paratíficas cuando por la existencia

58 No otra cosa es lo que sostiene César Cierco, quien defiende la necesidad general de una regulación específica en materia de vacunación pública, dada la complejidad de la materia, y su gran importancia social. en C. CIERCO, Vacunación, libertades individuales y Derecho público, cit.

59 En este sentido, Federico de Montalvo sostiene que sería necesario la aprobación de una norma legal que habilitara a las autoridades públicas a adoptar medidas de vacunación sistemática obligatoria puesto que, aunque la normativa actual sí habilitaría a las autoridades a imponer vacunas de forma puntual, por ejemplo para casos de epidemias, no sería suficiente para la adopción de un modelo basado en la obligatoriedad. F. DE MONTALVO JÄÄSKELÄINEN, "Rechazo a las políticas públicas de vacunación", cit., p. 234; También lo sostiene en "El paradigma de la autonomía en salud pública. ¿Una contradicción o un fracaso anticipado?: El caso concreto de la política de vacunación", Derecho y Salud, núm. 24, 2014, pp. 25-27.

60 Real Decreto sobre vacunación obligatoria y medios de extinción de la viruela, de 15 de enero de 1903.

61 Ley de Bases de Sanidad Nacional, de 25 de noviembre de 1944. 
de casos repetidos de estas enfermedades, estado epidémico del momento o previsible, se juzgue conveniente". Por último, la Ley dejó la puerta abierta a que las demás vacunas que en el futuro se desarrollaran pudieran imponerse de forma obligatoria por las autoridades sanitarias.

El modelo de la obligatoriedad se mantuvo hasta que en 1979 la OMS declaró erradicada la viruela, lo cual hizo que dejara de tener sentido vacunar obligatoriamente contra esta enfermedad. En consecuencia, en 1980, se promulgó la Ley 22/1980, la cual estableció que las vacunas contra la viruela y la difteria "podrían ser declaradas obligatorias", y que, respecto a las demás enfermedades, las vacunas podrían ser recomendadas o, en su caso, impuestas por las autoridades sanitarias. Desde entonces, ninguna vacuna ha vuelto a ser obligatoria ${ }^{62}$.

Ahora bien, quizás podría pensarse que si nuestro sistema de vacunación pudo ser obligatorio en los años anteriores a la entrada en vigor de la Constitución, fue por el reducido alcance de los derechos y las libertades que en aquellos momentos se reconocían. Nada más lejos de la realidad. Hoy en día, países de nuestro entorno, como Italia y Francia, han adoptado recientemente el modelo de la obligatoriedad sin que ello desvirtúe el carácter democrático de su Derecho. De hecho, de los sesenta y dos países en el mundo que establecen vacunas obligatorias, catorce de ellos son miembros de la Unión Europea ${ }^{63}$.

Los casos italiano y francés nos sirven de ejemplo de que puede adoptarse el modelo de vacunación obligatoria de forma totalmente compatible con un régimen pleno de derechos y libertades. En 2017, el Gobierno italiano promulgó un decreto-ley en el que declaró obligatorias diez vacunas. Asimismo, estableció que los padres deberían presentar la libreta de vacunación para poder inscribir a los niños de cero a seis años en guarderías y escuelas infantiles, así como multas administrativas para los casos de incumplimiento ${ }^{64}$.

62 César Cierco ha estudiado la consolidación de la vacunación obligatoria y el tránsito hacia el modelo recomendado o voluntario en nuestro país, en su libro: Vacunación, libertades individuales y Derecho público, cit, pp. 20-35.

63 En concreto, se prevén vacunas obligatorias en Bélgica, Bulgaria, Croacia, Chipre, República Checa, Eslovaquia, Eslovenia, Francia, Grecia, Hungría, Italia, Letonia, Malta, y Polonia. Katie Attwell ofrece una tabla con los 62 países que establecen una o más vacunas obligatorias, entre ellos los comunitarios, en K. ATWELL, et al., "Mandatory vaccination and no fault vaccine injury compensation schemes: An identification of country-level policies", Vaccine, núm. 37, 2019, p. 2846.

64 El Decreto-Ley fue convertido ese mismo año en la Legge 31 luglio 2017 n. 119, con modificazioni del Decreto-legge 7 giugno 2017, n. 73, recante disposizioni urgente in materia di prevenzione vaccinale. Las vacunas que estableció como obligatorias fueron las del tétanos, poliomielitis, hepatitis B, difteria, tos ferina, haemophilus influenzae tipo B, sarampión, pape-

ISSN: 1133-0937

DOI: https://doi.org/10.20318/dyl.2021.6111
DERECHOS Y LIBERTADES

Número 45, Época II, junio 2021, pp. 365-398 
Lo que provocó esta decisión fue un descenso notable de los niveles de vacunación que se relacionó con la aparición de algunos brotes epidémicos durante la segunda década del siglo pasado. En concreto, entre los años 2011 y 2016, la vacunación contra la poliomielitis se redujo en Italia en un $2.8 \%$, y la del sarampión en un 4.8\%. A consecuencia de ello, en 2016 se registraron 844 casos de sarampión, y 2.395 en 2017, de los cuales el 90\% afectaron a individuos no vacunados ${ }^{65}$. Para hacernos una idea de lo que supuso la epidemia de sarampión en Italia de 2017, baste señalar que ese país se convirtió ese año en el sexto del mundo con mayor propagación de la enfermedad, solo por detrás de la India, Nigeria, Paquistán, Ucrania y China.

La decisión de incrementar el número de vacunas obligatorias fue impugnada por la Región del Véneto, a través de un recurso presentado ante el Tribunal Constitucional. En sentencia de 24 de febrero de 2018, el Tribunal respaldó la adopción del modelo de la obligatoriedad, al estimarlo una medida necesaria para la "prevención de la difusión de enfermedades infecciosas" y para alcanzar el objetivo de la inmunidad de grupo ${ }^{66}$. Asimismo, observó que el legislador "ha intervenido en una situación en la que el instrumento de la persuasión carecía de eficacia" y que, por eso, "optó por la obligación" ${ }^{67}$. Quizás con esta última afirmación el Tribunal se refería a la experiencia piloto que se llevó a cabo en el Véneto, en donde en 2007 se suprimieron las vacunaciones obligatorias, y se reforzaron las campañas de concienciación ciudadana como medida compensatoria. El resultado del experimento fue que las vacunas se redujeron en un $5 \%$ en la región ${ }^{68}$.

Una enseñanza parecida suministra el caso francés. El modelo de vacunación pública en Francia anterior a 2018 establecía la obligatoriedad de tres vacunas (contra la difteria, el tétanos, y la poliomielitis). Pero aquel año el Gobierno fijó como obligatorias otras ocho más, siendo once el número total de vacunas obligatorias. Al igual que en el caso italiano, la medida fue acompañada de la imposición del requisito de la vacunación para escolarizar a los niños ${ }^{69}$.

ras, rubeola y varicela. W. RICCIARDI, et al., "Moving towards compulsory vaccination: The Italian experience", The European Journal of Public Health, vol. 28, núm. 1, p. 2.

65 Sobre esto véase C. TOLOSA TRIBIÑO, "El derecho a la salud y la vacunación en España y en el Derecho comparado", Anuario RANM, núm. 135, vol. 3, 2018, p. 327.

66 Sentencia $n^{\circ}$ 5/2018 del 22-11-2017.

67 El subrayado es nuestro.

68 C. SIGNORELLI, et al., "Childhood vaccine coverage in Italy after the new law on mandatory immunization", Annali di Igiene, núm. 30, vol. 4, 2018, p. 8.

69 Las ocho nuevas vacunas fueron contra las siguientes enfermedades: haemophilus influenzae tipo B, tos ferina, hepatitis B, sarampión, paperas, rubeola, meningococcus C y 
¿Por qué este cambio? La respuesta se encuentra en la suma de tres factores: el primero es que la situación de coexistencia entre vacunas obligatorias y voluntarias generaba la falsa percepción de que las primeras eran las realmente importantes, y que las otras eran opcionales o menos importantes. Una situación, por cierto, muy parecida a la eslovena, en la que el modelo mixto provocó que, durante el año 2016, el nivel de vacunación de las no obligatorias fuera mucho menor que el de las obligatorias ${ }^{70}$. Esto nos puede servir para comprobar los problemas que ocasiona este modelo mixto entre voluntariedad y obligatoriedad que, ante los extremos, quiere ofrecer una solución basada en la equidistancia entre un modelo y otro.

El segundo factor que propició el cambio es que Francia era uno de los países occidentales en los que las vacunas eran vistas con mayor desconfianza entre la población. De hecho, en 2015 el 41\% de los franceses encuestados se mostraba preocupado por la seguridad y eficacia de las vacunas ${ }^{71}$. A esto hay que añadir que, a consecuencia de una reducción de los niveles de vacunación -que se situaron por debajo del 84\% en 2018- hubo un brote de sarampión con 2.913 casos. El Primer Ministro, Édouard Philippe declaró que “des enfants meurent encore de la rougeole, dans la patrie de Pasteur ce n'est pas admisible" 72 .

¿Cuáles fueron los resultados del establecimiento de nuevas vacunas obligatorias en Francia? Por un lado, la opinión de la ciudadanía respecto a las vacunas mejoró notablemente, pues mientras que en 2015 los que pensaban que las vacunas eran inseguras representaban el $41 \%$, tres años más tarde eran el 23.7\%. Y respecto a la eficacia de las vacunas, si en 2015 eran el 17.3\%, en 2018 tan sólo representaban el 12.5\% ${ }^{73}$. Estos datos sirven como argumento para los que creen que la opinión desfavorable hacia las vacunas sólo puede -o incluso debe- ser combatida con vigorosas campañas de información y persuasión ciudadana. Ni la experiencia del Véneto, ni las en-

steptococcus pneumoniae. Véase J. WARD, et al., "Why France is making eight new vaccines mandatory", Vaccine, núm. 36, 2018, p. 1801.

70 En Eslovenia hay 9 vacunas obligatorias y 2 voluntarias. La diferencia en el número de vacunados es que, mientras la ratio de las obligatorias se sitúa entre el 90-95\%, el de las voluntarias ronda el 50\%. Sobre esto véase M. VRDELJA, et al., "From mandatory to voluntary vaccination: intention to vaccine in the case of policy changes", Public Health, núm. 180, 2020, pp. 1-3

71 R. COHEN, "Impact of mandatory extension on infant vaccine coverages: Promising preliminary results", Médecine et maladies infectieuses, núm. 49, 2019, p. 35.

72 S. ROY, “Matignon va render obligatoires onze vaccines", Le Figaro, 4 de julio de 2017.

73 D. LÉVY-BRUHL, et al., "Assessment of the impact of the extension of vaccination mandates on vaccine coverage after 1 year, France, 2019”, Euro Surveil, núm. 24, vol. 25, 2019, p. 4.

ISSN: 1133-0937

DOI: https://doi.org/10.20318/dyl.2021.6111
DERECHOS Y LIBERTADES

Número 45, Época II, junio 2021, pp. 365-398 
cuestas de opinión realizadas en Francia, aconsejan recurrir sólo a medidas de persuasión y fomento para incrementar los niveles de vacunación. Y por el otro lado, y como resultado evidente aún más importante, el número de vacunaciones también creció respecto a todas y cada una de las vacunas que pasaron a ser obligatorias ${ }^{74}$.

Para terminar con el caso francés, vale la pena señalar que, a propósito de la obligatoriedad de vacunar respecto a las tres primeras vacunas, se planteó una cuestión prioritaria de constitucionalidad por parte de una pareja que se negaban a vacunar a sus hijos. El Conseil Constitutionnel se pronunció en marzo de 2015 sobre la admisibilidad de la medida ${ }^{75}$. Frente al rechazo de los padres, el Conseil aseguró que es "lícito para el legislador definir una política de vacunación con el fin de proteger la salud individual y colectiva", con lo que la medida respondía adecuadamente al mandato constitucional de proteger la salud pública. Asimismo, concluyó que el sistema obligatorio no vulneraba ningún derecho o libertad garantizados por la Constitución, y que por ello la medida fue declarada conforme a Derecho ${ }^{76}$.

No hay que olvidar que, en el modelo de vacunación pública obligatoria, se genera una obligación legal que consiste en un hacer, en someterse a una intervención médica. Pero para que el establecimiento de este hacer, esta prestación de servicios, sea declarado conforme a Derecho, deberá respetar las exigencias que se derivan del conjunto del Ordenamiento jurídico para este tipo de obligaciones. Para saber qué exigencias son esas debemos mirar a la Constitución, la cual, si bien no establece los principios que deberán inspirar las prestaciones personales, sí lo hace respecto de las patrimoniales, las cuales pueden servir perfectamente para el caso que nos ocupa. Esos principios, referidos en el artículo 31.1 CE, son los de generalidad e igualdad, capacidad y no confiscatoriedad. Veamos, pues, qué exigen cada uno de ellos.

Lo que mandan los principios de generalidad e igualdad, es que la obligación de vacunar recaiga por igual sobre todos los individuos y que, por ello, todos estén sujetos de igual manera a la obligación. De acuerdo con él,

74 Las estadísticas concretas respecto a todas las vacunas pueden consultarse en ibidem, y en R. COHEN, “Impact of mandatory extension on infant vaccine coverages...", op.cit.

75 Décision Conseil constitutionnel, $\mathrm{n}^{\circ} 2015-458$ QPC de 20 de marzo de 2015.

76 En concreto, el Conseil constitutionnel aseguró que, al establecer vacunas obligatorias, el legislador "no ha vulnerado la exigencia constitucional de protección de la salud" garantizada por la Ley, y que las disposiciones impugnadas por los recurrentes "al no infringir ningún otro derecho o libertad garantizados por la Constitución, deben ser declarados conformes con la Constitución". Ibidem. 
todos los individuos, a menos que concurra una causa adecuada de exención, están obligados a vacunarse. Además de los españoles, también estarían obligadas las personas extranjeras que residan en España y que estén en posesión de la tarjeta sanitaria. No así, sin embargo, el resto de extranjeros que visiten temporalmente nuestro país, como turistas o viajeros. No hay que perder de vista que el fin último es el logro y mantenimiento de la inmunidad de grupo, y que, si se alcanzan cifras de vacunados incluso superiores a las mínimas que exige su activación, será posible compensar la llegada de turistas y visitantes no vacunados sin que por ello se haga peligrar la defensa inmunológica colectiva ${ }^{77}$.

Ahora bien, hay que señalar que el hecho de que los individuos estén obligados vacunarse no significa que todos vayan a tener que hacerlo. La obligación sólo sería exigible a quienes cumplieran con una serie de requisitos técnicos que, por supuesto, deberían acompañar a la medida, requisitos que son los que ya están establecidos hoy en día para preservar la salud de las personas y que provienen de la larga experiencia en vacunación, la cual aconseja, por ejemplo, administrar determinadas vacunas sólo cuando los pacientes alcancen determinada edad y en el número de dosis indicada -por ejemplo, la vacuna contra la poliomielitis se administra al cumplir los 2, 4 y 11 meses, y a los 6 años-, verificar el estado de vacunación previo antes de inocular una dosis, vacunar de la gripe sólo a embarazadas y a personas mayores de 65 años, etcétera ${ }^{78}$.

El párrafo anterior nos conduce a los principios de capacidad y no confiscatoriedad, que exigen, en definitiva, que cuando se impone legalmente una obligación tenga en cuenta, en cada caso, las circunstancias de los individuos. Uno de los argumentos más usados para desaconsejar la vacunación obligatoria es que se trata de una medida masiva, y que en consecuencia comportaría una desatención de los casos particulares. Todo lo contario de lo que exigen estos dos principios: atender al estado de salud de cada individuo a fin de velar en todo momento por su salud, evitando que pueda perjudicársele. Se trata, por lo tanto, de respetar el viejo principio de primum

77 Tal y como ha afirmado, Giubilini, "the more the rate of international travels intensifies, the less meaningful and useful herd immunity becomes as a preventive measure". A. GIUBILINI, The Ethics of Vaccination, cit., p. 19.

78 Para más información sobre vacunación adulta, véase https://www.mscbs.gob.es/profesionales/saludPublica/prevPromocion/vacunaciones/docs/Vacunacion_poblacion_adulta.pdf. Y para más información sobre vacunación infantil, véase $h t t p s: / / w w w . m s c b s . g o b . e s / p r o f e s i o n a l e s / s a l u d-$ Publica/prevPromocion/vacunaciones/docs/CalendarioVacunacion_Todalavida.pdf

ISSN: 1133-0937

DOI: https://doi.org/10.20318/dyl.2021.6111
DERECHOS Y LIBERTADES

Número 45, Época II, junio 2021, pp. 365-398 
non nocere, el cual aconseja establecer un régimen de exenciones por razones médicas, que son, como ya vimos, las que se refieren a personas inmunodeprimidas, recién nacidos, pacientes de quimioterapia, de trasplantes de órganos, de leucemia, etcétera, obviamente no aptos para la vacuna. También habrá que velar por el respeto al consentimiento informado, es decir, que el personal sanitario informe a las ciudadanía de acuerdo con lo establecido por la Ley, y recabe su consentimiento para llevar a cabo el acto médico ${ }^{79}$.

Esto nos lleva a que sería preciso organizar un régimen sancionador para los casos de incumplimiento, en tanto que estamos hablando de una obligación legal. Las consecuencias que deberían seguirse en los supuestos de no cumplir con la obligación de vacunarse son uno de los aspectos más difíciles y controvertidos que acarrea esta obligación. Algunos, como Jessica Flanigan, proponen que habría que excluir a los no vacunados de determinados servicios públicos, como por ejemplo la escolarización a los niños. La misma autora es también partidaria de aplicar restricciones en el empleo respecto a sectores como la sanidad o el cuidado de personas, a fin de mitigar la transmisión de enfermedades, al igual que de establecer multas y destinaría lo recaudado a financiar campañas de concienciación a favor de la vacuna. Flanigan hasta llega al punto de imputar a los no vacunados la responsabilidad de los daños producidos. Siempre que fuera posible rastrear el origen de un brote y determinar que el causante fue un individuo no vacunado, los perjudicados deberían poder demandarle ${ }^{80}$.

A nuestro modo de ver, la medida más adecuada para abordar los supuestos de incumplimiento debería ser, en primer lugar, la imposición de una multa administrativa. César Cierco, ha alertado de que el problema de establecer multas es que podrían llegar a convertirse en una especie de "bula-antivacunación", que permitieran sortear la obligación con el simple abono del importe a los más afortunados ${ }^{81}$. Ahora bien, como señala este autor, la finalidad de una multa no es penalizar sin más, sino vencer la resistencia de los individuos, razón por la cual, de adoptarse esta medida, debería im-

79 Que la vacuna sea obligatoria no dispensa al personal sanitario de informar al obligado y de recabar su consentimiento, según lo dispuesto en las leyes para todo acto médico, pues una cosa es el consentimiento en relación a un acto médico en sí, y otra las razones por las que la persona decide prestar su consentimiento, que pueden ser muchas y muy variadas, y que al médico no le son en absoluto pertinentes.

80 Sobre esto véase J. FLANIGAN, “A Defence of Compulsory Vaccination”, cit., pp. 20-22.

81 C. CIERCO, Vacunación, libertades individuales y Derecho público, cit., p. 48-49. 
ponerse de forma periódica, con una cantidad incluso creciente en el tiempo. A la multa podría acompañarle además la llamada "vacuna-condición", esto es, convertir la vacuna en un requisito para, por ejemplo, poder escolarizar a los niños.

De hecho, no otra cosa es lo previsto para estos supuestos en Italia y en Francia: en el país transalpino se establecen multas administrativas que pueden ir de los cien a los quinientos euros para los casos de incumplimiento, y se exige a los padres que certifiquen que sus hijos cumplen con el calendario de vacunación para poder escolarizarlos, mientras que en Francia sólo se exige esta segunda medida ${ }^{82}$. Pero es que son precisamente esas medidas las que ya fueron adoptadas en nuestro país por el Real Decreto de 1903, el cual estableció, en su artículo $8^{\circ}$, que la "inobservancia o falta de puntualidad será corregida con multas gubernativas", y que los padres debían presentar el certificado de vacunación de sus hijos siempre que la autoridad lo exigiera (artículo $9^{\circ}$ ), ya que, sin el certificado en regla, "no se concederá ingreso en Escuela pública, Colegio ó Liceo particular" (artículo 13²) ${ }^{83}$.

En cualquier caso, la compulsión, o vacunación forzosa, debería ser una medida subsidiaria de las anteriores, por ser más gravosa, y quedar reservada como último recurso para los supuestos más graves. Aunque es cierto que la materia correspondiente al régimen sancionador se presta a una amplia especulación sobre la mayor o menor eficacia de las diversas medidas, bastan las experiencias italiana y francesa para comprobar que la compulsión no sería necesaria, y que la simple amenaza de una multa, junto con la "vacuna-condición", son suficientes para incrementar notablemente el número de individuos vacunados. Así lo ha sostenido, tras examinar este asunto, el filósofo del Derecho Roland Pierik, el cual asegura que la experiencia prueba que "mandatory vaccination through fines provides enough an incentive to generate sufficient vaccination rates to maintain robust herd immunity", y

82 En Italia, el artículo 4 del Decreto-legge de 7 de junio de 2017 establece sanciones administrativas que pueden ir de los cien a los quinientos euros, y el Decreto legislativo de 16 de abril de 1994, Testo Unico delle disposizioni legislative vigente in materia di istruzione, delative alle scuole di ogni ordine e grado, prevé, en su artículo 100, que "l'amisssione alla scuola materna è subordinata al possesso del requisito dell'età (...) e alla presentazione della certificazione delle vaccinazoni di cui all'articolo 117". En Francia, el Décret núm. 2018-42 de 25 de enero de 2018 relatif à la vaccination obligatoire estableció, en su artículo 2, que la admisión de los niños a escuelas y centros de enseñanza estaría subordinado a la presentación del certificado de vacunación.

83 Real Decreto sobre vacunación obligatoria y medios de extinción de la viruela, de 15 de enero de 1903. 
que, por ello, "only when this is not the case a more intrusive policies can be considered" 84 .

\section{4. ¿SON CONVINCENTES LAS OBJECIONES?}

Una de las razones a las que se apela para oponerse a la vacunación obligatoria es que la obligatoriedad viola el derecho a la integridad física. Mark Navin, quien ha dedicado un libro a analizar las objeciones que plantean quienes rechazan la vacunación, asegura que "vaccines refusers may insist that coercive vaccination violates bodily integrity, which is something that the state may not do" ${ }^{\prime 5}$. Por su parte, Giubilini señala sobre todo a los "nozickian libertarians" como aquellos que afirman que "bodily autonomy is a fundamental liberty, and therefore the state is not justified in violating bodily autonomy to promote a good that is valuable for the community" 86 .

"Yo no me quiero vacunar y nadie puede obligarme, porque la Constitución protege mi negativa". Esto es lo que parecen pensar algunos, que consideran que la imposición de vacunas es incompatible con el derecho a la integridad física que reconoce en el artículo 15 la Constitución española. Nuestro Tribunal Constitucional (a partir de ahora TC), en la STC 120/1992, de 21 de septiembre, señaló que "este derecho constitucional (el derecho a la integridad física reconocido en el artículo 15) resultará afectado cuando se imponga a una persona asistencia médica en contra de su voluntad", a lo que añadió que "esa asistencia médica coactiva constituirá limitación vulneradora del derecho fundamental, a no ser que tenga justificación constitucional". Parece, pues, que cabría apoyarse en el derecho a la integridad física para justificar la resistencia a imponer una vacunación.

Ahora bien, hay que tener en cuenta que los derechos no son absolutos, y que deben ser armonizados entre sí. Como recordó el Tribunal Superior de Justicia de Andalucía, en relación a un caso en el que declaró ajustada a Derecho la decisión de vacunar forzosamente a los hijos de unos padres que se negaban a hacerlo, "la convivencia en un Estado social y democrático de Derecho supone, no sólo el respeto a los derechos fundamentales a título individual, sino también que su ejercicio no menoscabe el derecho del resto de

\footnotetext{
84 R. PIERIK, “Mandatory Vaccination...”, cit., p. 389.

85 M. NAVIN, Values and Vaccine Refusal. Hard Questions in Ethics, Epistemology, and Health Care, Routledge, Nueva York, 2016, pp. 183 y ss.

86 A. GIUBILINI, The Ethics of Vaccination, cit., p. 104.
} 
la sociedad que se rige por unas pautas de conducta que persiguen el interés general" 87 .

Nos encontramos en un escenario en el que la salud de todos está expuesta a un grave riesgo, lo cual es razón suficiente para limitar el derecho a la integridad física de los individuos. Salus populi suprema lex est. Y vacunar a determinados individuos a cambio de garantizar protección colectiva frente a enfermedades infecciosas, es una medida perfectamente armonizable con la salvaguarda del derecho a la integridad física.

Es importante señalar por ello que el propio TC ha indicado explícitamente que el derecho a la integridad física no es un derecho absoluto, y que puede ceder si es ante razones de interés general, debidamente establecidas y justificadas por la Ley. Y, claro está, la salud pública es una de esas razones. En la STC 207/1996, el TC explicitó qué exigencias debería cumplir cualquier medida que afectara a un derecho fundamental, así como, y de manera más concreta, los que afectaran al derecho a la integridad física ${ }^{88}$. Veamos esto.

En relación con las medidas restrictivas de derechos fundamentales, el TC sostuvo que deberían cumplir con las exigencias generales de razonabilidad y proporcionalidad, que se concretan en las condiciones de idoneidad -que la medida sea susceptible de conseguir el objetivo propuesto-, de necesidad -que no exista otra medida menos restrictiva e igual de eficaz-, y de proporcionalidad en sentido estricto -que, en relación con los bienes en colisión, la medida genere más beneficios que perjuicios ${ }^{89}$. Pues bien, parece claro que la vacunación obligatoria cumpliría con las tres condiciones, porque permitiría alcanzar y mantener la inmunidad de grupo, como han demostrado las experiencias italiana y francesa, y cumplir así con el juicio de idoneidad. Ya hemos visto que no habría otra medida menos gravosa que permitiera asegurar la inmunidad de grupo, por lo que también cumpliría con la condición de necesidad. Por último, cumpliría con la exigencia de proporcionalidad, porque a cambio de una afectación menor a la integridad física, lograríamos activar el escudo de la inmunidad de grupo ${ }^{90}$.

87 Véase el Fundamento de Derecho cuarto de la STSJ de Andalucía 8545/2013, de 22 de julio.

88 STC 207/1996, de 16 de diciembre.

89 Sobre el principio de proporcionalidad como guía para la resolución de los conflictos de derechos, véase M. J. AÑ́́N ROIG, ¿Hay límites a la regresividad de derechos sociales?, Derechos y Libertades, núm. 34, 2016, pp. 79 y ss.

90 Juan José González López ha sostenido que la imposición obligatoria de la vacunación a los menores no maduros es susceptible de superar el juicio de proporcionalidad, 
Antes de proseguir, sin embargo, valdría la pena descartar, a propósito del juicio de necesidad, la reclusión o confinamiento domiciliario como medida alternativa y menos restrictiva para los que se negasen a la vacunación obligatoria. Es cierto que la reclusión no permitiría alcanzar la inmunidad de grupo, pero es que ni siquiera sería menos gravosa que la propia compulsión. En la citada STSJ de Andalucía 8545/2013, el Tribunal tuvo que valorar si la vacunación forzosa cumplía el juicio de proporcionalidad, porque la parte recurrente entendía que era más razonable el aislamiento domiciliario voluntario. El Tribunal concluyó que "la Sala no llega a vislumbrar por qué sería más proporcional tal aislamiento, ya que el mismo incidiría sobre el derecho fundamental de libre circulación de las personas, tendría una duración incierta (dado que habría que esperar para su finalización a la erradicación total del brote) y su cumplimiento sería de difícil control por parte de la Administración" ${ }^{91}$.

Además de las condiciones a cumplir por cualquier medida que afecte a un derecho fundamental, el TC también detalló, en la misma STC 207/1996, las tres exigencias que debería acreditar toda medida que restringiera concretamente el derecho a la integridad física: en primer lugar, que "en ningún caso podrá acordarse la práctica de una intervención corporal cuando pueda suponer (...) un riesgo o quebranto para la salud". En segundo lugar, que en cualquier caso la intervención sea llevada a cabo "por personal sanitario, que deberá ser personal médico especializado en el supuesto de intervenciones graves que lo requieran"; en tercer lugar, que la intervención deberá efectuarse "con respeto a la dignidad de la persona, sin que pueda en ningún

y representa una alternativa legislativa constitucionalmente admisible. Cf. J. J. GONZÁLEZ LÓPEZ, "Análisis de la admisibilidad constitucional de la vacunación obligatoria de menores", Derecho y Salud, vol. 26, 2016, p. 167. En el mismo sentido, María Martín Ayala defiende la instauración de un calendario vacunal único y obligatorio para todo el Estado. M. MARTÍN AYALA, en "Vacunación infantil", Derecho y Salud, vol. 24, núm. extra. 1, 2014, pp. 182-192.

91 También ha defendido que la reclusión domiciliar es más gravosa que la vacunación forzosa STAN BLOCK, quien afirma que, "during a possible vaccine-preventable illness, the child must not only be isolated from one's office, but also from day care, school, and susceptible siblings". Véase del autor "The Pediatrician's Dilemma: Refusing the Refusers of Infant Vaccines", Journal of Law, Medicine E Ethics, 2015, pp. 650. En la misma línea JESSICA FLANIGAN ha señalado que "quarantine is a limitation on a potentially contagious person's (...) the cost to the individual of preventing transmission is typically higher than in the case of non-vaccination because it involves isolation rather than a unwanted injection or medication", y añade que, por otro lado, "the risks posed by contagious people who are subject to quarantine are also higher". De la autora, “A Defence of Compulsory Vaccination”, cit., p. 13. 
caso constituir, en sí misma o por la forma de realizarla, un trato inhumano o degradante".

No se ven grandes problemas para que la vacunación obligatoria cumpliera con estos tres requisitos, pues el personal sanitario sería el encargado de vacunar a los individuos con todas las garantías sanitarias, del mismo modo que, por cierto, se hace hoy en día; y la dignidad de las personas se vería respetada absolutamente. También cumpliría con el requisito de no poner en riesgo la salud de los individuos. Hay que recordar que este modelo iría acompañado de una serie de requisitos técnicos, y de un sistema de exenciones, de tal modo que no se vacunaría a los individuos para los que la vacunación pudiera comportar problemas de salud, ya fuera por el estado en que se encuentren, ya por estar en situaciones contraindicadas con la administración de vacunas.

De hecho, no otra cosa es lo que se hace en Italia y en Francia. En el país transalpino, el artículo 1.3 del Decreto-Legge citado establece que las vacunaciones "possono essere omesse o differite solo in caso di accertato pericolo per la salute, in relazione a specifiche condizioni cliniche documentate, attestate dal medico di medicina generale o dal pediatra di libera scelta". Mientras que en Francia, el Code de la santé publique establece, en su artículo L3111-2, que "les vaccinations suivantes sont obligatoires, sauf contre-indication médicale reconnue, dans des conditions d'âge déterminées par décret en Conseil d'Etat, pris après avis de la Haute Autorité de santé ".

César Cierco ha señalado a la objeción de conciencia como una de las grietas o puntos más débiles del modelo de la obligatoriedad, por cuanto que se la invoca para resolver la colisión entre la vacunación obligatoria y la libertad de pensamiento, de conciencia y de religión ${ }^{92}$. Sin embargo, aunque $a$ priori pudiera verse dicha objeción como algo inherente al establecimiento de cualquier obligación legal que imponga una prestación de servicios, así la de prestar el servicio militar, lo cierto es que, examinado de cerca el asunto, no parece haber excesivas buenas razones para habilitar esta suerte de objeción de conciencia vacunal.

92 C. CIERCO, Vacunación, libertades individuales y Derecho público, cit., p. 93. Para saber más sobre el debate entre la objeción de conciencia y la vacunación obligatoria, pueden verse D. SALMON y A. SIEGEL, "Religious and Philosophical Exemptions from Vaccination Requirements and Lessons Learned from Conscientious Objectors from Conscription", Public Health Reports, vol. 116, 2001, pp. 289-295, y S. CLARKE, et al., "Conscientious Objection to Vaccination", Bioethics, núm. 31, vol. 3, 2017, pp. 155-161. 
En primer lugar, porque no está nada claro que la vacunación obligatoria colisione con esas libertades. Como ha apreciado César Cierco, "los vínculos entre la vacunación y la libertad de pensamiento, conciencia y religión distan de ser claros" a efectos de ser tenidos como motivo de exoneración ${ }^{93}$. Pierik cree por su parte que la libertad religiosa y de creencias no deben ser vistas, en ningún caso, como límites a la vacunación obligatoria, y no considera por ello que deba habilitarse una objeción de conciencia por razones no médicas ${ }^{94}$. Tampoco la Comisión Europea de Derechos Humanos entendió que la vacunación obligatoria representara una interferencia en el ejercicio de tales libertades. En 1998, este órgano tuvo que decidir si la vacunación obligatoria era contraria a la libertad de pensamiento, conciencia y religión protegida por el artículo 9 del Convenio Europeo de Derechos Humanos y concluyó que "the obligation to be vaccinated, as laid down in the legislation at issue, applies to everyone, whatever their religion or personal creed". En consecuencia, consideraba que "there has been no interference with the freedom protected by art. 9 para 1 of the Convention" ${ }^{95}$. Asimismo, merece la pena señalar que, aunque no pueda descartarse que en un futuro aparezca una religión que rechace explícitamente la vacunación, de momento no la hay y, en todo caso, sería necesaria cierta plausibilidad intelectual respecto a las razones no médicas que impidan vacunar.

A esto hay que añadir que las exenciones por razones no médicas serían contrarias al principio de generalidad e igualdad, que ya vimos que exige que todos los individuos estén igualmente obligados a vacunarse ${ }^{96}$. Pierik incluso llega a afirmar que las exenciones por motivos religiosos discriminarían a los ateos, porque serían ellos, como casi siempre, los únicos que tendrían que soportar las cargas que exige la vida en comunidad ${ }^{97}$. Por su parte, Flanigan rechaza de pleno las exenciones por razones no médicas, porque entiende que "religious freedom and rights of informed consent no not entitle non-vaccinators to harm innocent bystanders", es decir, que esos derechos no legitimarían al objetor a, en definitiva, perjudicar a terceros ${ }^{98}$.

Por último, otra razón que desaconsejaría abrir cauce a una objeción de conciencia, es que, de hacerlo, se frustraría el objetivo último de lograr y

93 C. CIERCO, Vacunación, libertades individuales y Derecho público, cit., p. 93.

94 R. PIERIK, “Mandatory Vaccination...", cit., pp. 389-390.

95 Decisión de 15 de enero de 1998, Carlo Boffa y otros c. San Marino, p. 34.

96 Así lo entiende al respecto A. GIUBILINI en The Ethics of Vaccination, cit., pp. 118-119.

97 R. PIERIK, "Mandatory Vaccination...", cit., p. 392.

98 J. FLANIGAN, “A Defence of Compulsory Vaccination”, cit., p. 17-19. 
mantener la inmunidad de grupo, lo cual haría que todo el modelo de vacunación obligatoria careciera de sentido. Esta ha idea ha sido defendida por Thomas May y Ross Silverman, los cuales aseguran que el modelo más efectivo de lograr este objetivo es denegar todas las exenciones no médicas ${ }^{99}$. Si abrimos la puerta a la objeción de conciencia, el modelo de la obligatoriedad se tornaría ineficaz y, lo que a priori sería una obligación, a posteriori no lo sería; y no lo sería porque la objeción representaría un camino que, en apariencia restringido, permitiría que todo ciudadano que alegara razones no médicas, quedara dispensado de vacunarse. Quizás sea precisamente por este motivo por lo que ni el sistema francés ni el italiano han contemplado la “objeción de conciencia vacunal", y sólo permiten la exención por razones médicas ${ }^{100}$.

Es interesante comprobar el cambio de rumbo que están tomando algunos Estados de los EE.UU. en relación con este asunto. En el país americano, en donde existe la vacunación obligatoria, todos los Estados prevén exenciones por razones médicas, 48 de ellos por razones religiosas y solo 20 por creencias personales o de índole filosóficas. Y el incremento reciente en el número casos en los que se apela a razones no médicas para evitar la vacunación está llevando a que los estados de la federación traten de limitar las exenciones no médicas o, cuanto menos, a restringir y dificultar su tramitación. Un buen ejemplo de ello es el cambio de políticas en el Estado de California, en donde, a consecuencia del brote de sarampión de Disneyland en 2015, se decidió eliminar totalmente las exenciones no médicas ${ }^{101}$.

Cabe afirmar, por lo tanto, que no solo el modelo de vacunación pública obligatoria tendría perfecta cabida en nuestro Ordenamiento jurídico, por ser perfectamente armonizable con el régimen de derechos y libertades

99 T. MAY, y R. SILVERMAN, "Free-Riding, Fairness and the Rights of Minority Groups in Exemption from Mandatory Childhood Vaccination", Human Vaccines, núm. 1, vol. 1, 2005, p. 14.

100 A pesar de que hay buenas razones para adoptar el modelo de vacunación pública obligatoria y para rechazar la objeción de conciencia vacunal, esto no nos debe llevar tan lejos como para pensar, como hacen algunos, que es legítimo impedir que las personas puedan expresarse libremente sobre esta materia. También hay buenas razones para establecer impuestos, por ejemplo, pero no parece tanto que las haya para evitar que pueda ser discutida su conveniencia o idoneidad.

101 Sobre esto, véase C. CONSTABLE, et al., "Rising rates of vaccine exemptions: Problems with current policy and more promising remedies", Vaccine, núm. 32, 2014, pp. 1793-1797, y R. PIERIK, “Mandatory Vaccination...”, cit., p. 395. 
constitucionales, sino que existen buenas razones que aconsejan adoptarlo, ya que no sólo es el único que permite alcanzar y mantener la inmunidad de grupo, sino que es el que mejor respeta los aspectos éticos de la vacunación.

\author{
Pol Cuadros Aguilera \\ Universitat de Lleida \\ Facultad de Derecho, Economía y Turismo \\ Calle Jaume II, 73 \\ 25001 Lleida. \\ e-mail:pol.cuadros@udl.cat
}

